\title{
Cutting force-based adaptive neuro-fuzzy approach for accurate surface roughness prediction in end milling operation for intelligent machining
}

\author{
Ibrahem Maher • M. E. H. Eltaib • Ahmed A. D. Sarhan • \\ R. M. El-Zahry
}

removal operations encountered in industrial processes. Product quality is a critical issue as it plays a vital role in how products perform and is also a factor with great influence on manufacturing cost. Surface roughness usually serves as an indicator of product quality. During cutting, surface roughness measurement is impossible as the cutting tool is engaged with the workpiece, chip and cutting fluid. However, cutting force measurement is easier and could be used as an indirect parameter to predict surface roughness. In this research work, a correlation analysis was initially performed to determine the degree of association between cutting parameters (speed, feed rate, and depth of cut) and cutting force and surface roughness using adaptive neuro-fuzzy inference system (ANFIS) modeling. Furthermore, the cutting force values were employed to develop an ANFIS model for accurate surface roughness prediction in CNC end milling. This model provided good prediction accuracy ( $96.65 \%$ average accuracy) of surface roughness, indicating that the ANFIS model can accurately predict surface roughness during cutting using the cutting force signal in the intelligent machining process to achieve the required product quality and productivity.

I. Maher $(\bowtie)$. A. A. D. Sarhan

Centre of Advanced Manufacturing and Material Processing,

Department of Mechanical Engineering, University of Malaya,

50603 Kuala Lumpur, Malaysia

e-mail: ibrahemmaher@eng.kfs.edu.eg

I. Maher

Department of Mechanical Engineering, Faculty of Engineering, Kafrelsheikh University, Kafrelsheikh 33516, Egypt

M. E. H. Eltaib · A. A. D. Sarhan · R. M. El-Zahry Department of Mechanical Engineering, Faculty of Engineering, Assiut University, Assiut 71516, Egypt

forces $\cdot$ Surface roughness $\cdot \mathrm{CNC} \cdot$ ANFIS

1 Introduction

Machining processes are fundamentally complex, nonlinear, multi variate, and often subjected to various unknown external disturbances. A machining process is usually performed by a skilled operator who uses decision-making capabilities based on the intuition and rules of thumb gained from experience. This process is not accurate enough and in many cases product faults occur. For this reason and to realize highly productive and flexible machining, a reliable, automated machining system with intelligent functions (intelligent machining) is needed [1, 2]. Figure 1 depicts the concept of an intelligent $\mathrm{CNC}$ machine. Intelligent $\mathrm{NC}$ machine tools have three feedback loop levels for intelligent functions. Among the intelligent functions, cutting force monitoring is an important issues, as it can tell the limits of cutting conditions, workpiece surface quality, and tool wear, as well as detect and prevent tool breakage and chatter, compensate tool deflections, and optimize machining processes through a model-based adaptive control system and other process information, which are indispensable for process feedback control [3-6].

In this research work, cutting force is used to predict surface quality during cutting in an end milling process. Surface quality plays a vital role in milled surfaces by significantly improving fatigue strength, corrosion resistance, and creep life. Moreover, surface quality affects several functional attributes of parts, such as contact causing surface friction, wear, light reflection, heat transmission, ability of distributing and holding lubricant, coating, and resisting fatigue $[7,8]$. 
Fig. 1 System configuration of the intelligent $\mathrm{NC}$ machine too [1]

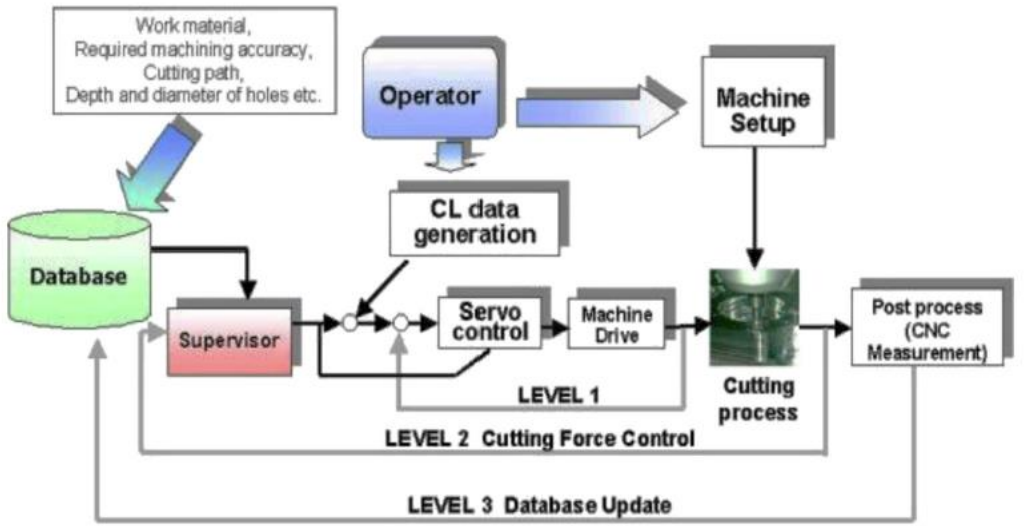

To achieve higher levels of surface quality, correlation modeling of cutting force and surface roughness is required [9-11]. Modeling based on cutting force and surface roughness data is accomplished by soft computing tools [12-14]. Soft computing techniques are useful when exact mathematical information is not available and these differ from conventional computing in that they are tolerant of imprecision, uncertainty, partial truth, approximation, and met heuristics [15, 16]. ANFIS is one of the soft computing techniques that play a significant role in input-output matrix relationship modeling. It is used when subjective knowledge and expert suggestions are significant to defining objective function and decision variables. ANFIS is ideal to predict surface roughness based on input variables due to the nonlinear condition in the machining process [17-21].

As a conclusion of the above review, the aim of the present work is to investigate the use of cutting forcebased ANFIS modeling for accurate surface roughness prediction in end milling operation for intelligent machining.

\section{Experimental setup}

The experimental setup is shown in Fig. 2. The experiments speed steel four-flute end milling cutter with a diameter of $7 / 16$ in $(11.1 \mathrm{~mm})$ was used for dry machining slots of Brass $(60 \mathrm{Cu} 40 \mathrm{Zn})$ blocks under specific machining conditions, as shown in Table 1. These machining conditions were selected based on the tool maker's recommendations. Brass material with Vickers hardness of 125 and chemical composition of $60 \%$ Copper and $40 \%$ Zinc was used as workpiece material with $40 \times 40 \times 20 \mathrm{~mm}$ dimensions.

The surface roughness $(R a)$ was measured with a stylusbased profilometer (Surtronic 3+, accuracy of $99 \%$ ). The average surface roughness was calculated for three different measurements under the same conditions with a sampling were performed using a $\mathrm{CNC}$ end milling machine. A high-

Fig. 2 Experimental setup

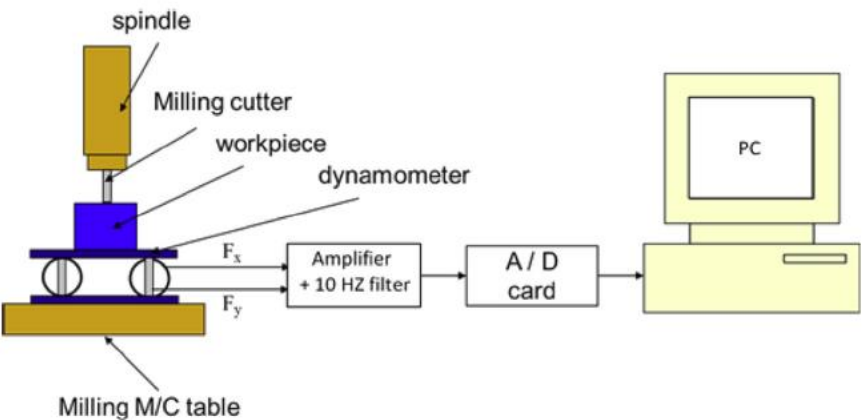


Table 1 Cutting parameters levels

\begin{tabular}{llllllll}
\hline Cutting parameters & Unit & Symbol & Level 1 & Level 2 & Level 3 & Level 4 & Level 5 \\
\hline Spindle speed & $\mathrm{rpm}$ & $n$ & 750 & 1000 & 1250 & 1500 & 1750 \\
Feed rate & $\mathrm{mm} / \mathrm{min}$ & $f$ & 50 & 100 & 150 & 200 & 250 \\
Depth of cut & $\mathrm{mm}$ & $t$ & 0.3 & 0.5 & 0.7 & & \\
\hline
\end{tabular}

length of $L c=2.5 \mathrm{~mm}$ at a specific area of the workpiece. The measurements' direction was parallel to the cutting direction and perpendicular to the lay of surface anomalies. On the other hand, the cutting forces were measured using a strain gaugebased analogue dynamometer. The analogue values obtained from the dynamometer were amplified, filtered with $10 \mathrm{~Hz}$ low bass filter and recorded on a computer using a 12 bit analogue-to-digital converter.

\section{Experimental results and ANFIS modeling}

The measured cutting forces and surface roughness shown in Table 2 were used as the training data set to build the ANFIS model. Five network layers were used by ANFIS to perform the following fuzzy inference steps as shown in Fig. 3: layer 1-input fuzzification, layer 2-fuzzy set database construction, layer 3-fuzzy rule base construction, layer 4 -decision making, and layer 5-output defuzzification [22-24].
To explain this model simply, two rules and two linguistic values for each input variable are suggested.

Layer 1 The output of the node is the degree to which the given input satisfies the linguistic label associated to this node. Usually, bell-shaped membership functions are chosen to represent the linguistic terms because the relationship between the cutting parameters and surface roughness is not linear (Fig. 4a).

First parameter membership functions

$A_{i}(x)=\exp \left[-0.5\left(\left(x-a_{i 1}\right) / b_{i 1}\right)^{2}\right]$

Second parameter membership functions

$B_{i}(y)=\exp \left[-0.5\left(\left(y-a_{i 2}\right) / b_{i 2}\right)^{2}\right]$

where $a_{\mathrm{i} 1}, a_{\mathrm{i} 2}, b_{\mathrm{i} 1}$, and $b_{\mathrm{i} 2}$ are the parameter set.

As the values of these parameters change, the bell-shaped functions vary accordingly, as shown in Fig. 4b, thus

Table 2 Measured cutting forces and surface roughness (training data set)

\begin{tabular}{|c|c|c|c|c|c|c|c|c|c|c|c|}
\hline \multicolumn{2}{|l|}{$n$ (rpm) } & \multicolumn{2}{|l|}{750} & \multicolumn{2}{|l|}{1000} & \multicolumn{2}{|l|}{1250} & \multicolumn{2}{|l|}{1500} & \multicolumn{2}{|l|}{1750} \\
\hline $\mathrm{f}(\mathrm{mm} / \mathrm{min})$ & $\mathrm{t}(\mathrm{mm})$ & $\mathrm{F}(\mathrm{N})$ & $\mathrm{Ra}(\mu \mathrm{m})$ & $\mathrm{F}(\mathrm{N})$ & $\mathrm{Ra}(\mu \mathrm{m})$ & $\mathrm{F}(\mathrm{N})$ & $\mathrm{Ra}(\mu \mathrm{m})$ & $\mathrm{F}(\mathrm{N})$ & $\operatorname{Ra}(\mu \mathrm{m})$ & $\mathrm{F}(\mathrm{N})$ & $\mathrm{Ra}(\mu \mathrm{m})$ \\
\hline \multirow[t]{3}{*}{50} & 0.3 & 25.61 & 1.1 & 15.26 & 0.96 & 10.63 & 1.18 & 11.31 & 0.6 & 7.21 & 0.84 \\
\hline & 0.5 & 39.81 & 1.36 & 30.53 & 1.12 & 14.87 & 1.6 & 19.80 & 0.82 & 12.04 & 0.82 \\
\hline & 0.7 & 50.61 & 1.9 & 37.54 & 1.36 & 22.63 & 1.08 & 27.59 & 1.02 & 12.73 & 1.54 \\
\hline \multirow[t]{3}{*}{100} & 0.3 & 33.42 & 1.28 & 17.03 & 1.02 & 10.82 & 1.18 & 14.87 & 0.86 & 20.52 & 0.98 \\
\hline & 0.5 & 64.88 & 2.06 & 44.05 & 1.44 & 21.21 & 1.3 & 30.48 & 1.02 & 45.45 & 1.16 \\
\hline & 0.7 & 106.83 & 2.22 & 62.94 & 1.78 & 25.50 & 1.14 & 40.61 & 1.24 & 73.36 & 1.22 \\
\hline \multirow[t]{3}{*}{150} & 0.3 & 31.14 & 1.42 & 21.21 & 1.54 & 18.44 & 1.24 & 20.52 & 1.32 & 31.62 & 1.1 \\
\hline & 0.5 & 81.84 & 2.63 & 50.22 & 1.54 & 22.80 & 1.34 & 37.01 & 1.36 & 70.04 & 1.26 \\
\hline & 0.7 & 113.81 & 2.96 & 78.49 & 2.24 & 32.53 & 1.22 & 52.40 & 1.38 & 92.44 & 1.62 \\
\hline \multirow[t]{3}{*}{200} & 0.3 & 30.41 & 1.54 & 18.38 & 1.16 & 22.63 & 1.26 & 25.24 & 1.56 & 39.56 & 1.32 \\
\hline & 0.5 & 78.77 & 3.5 & 61.62 & 2.28 & 30.41 & 1.5 & 49.24 & 1.56 & 86.56 & 1.62 \\
\hline & 0.7 & 144.90 & 3.52 & 102.53 & 2.64 & 42.64 & 1.44 & 56.82 & 1.4 & 104.24 & 1.6 \\
\hline \multirow[t]{3}{*}{250} & 0.3 & 70.21 & 1.82 & 23.02 & 1.58 & 25.50 & 1.66 & 35.47 & 1.32 & 56.65 & 1.48 \\
\hline & 0.5 & 58.67 & 2.5 & 61.59 & 2.96 & 28.32 & 1.38 & 54.08 & 1.26 & 106.96 & 1.74 \\
\hline & 0.7 & 190.80 & 5.5 & 106.78 & 3.14 & 38.18 & 1.62 & 49.52 & 1.42 & 107.94 & 1.56 \\
\hline
\end{tabular}


Fig. 3 ANFIS architecture for two-input Sugeno fuzzy model

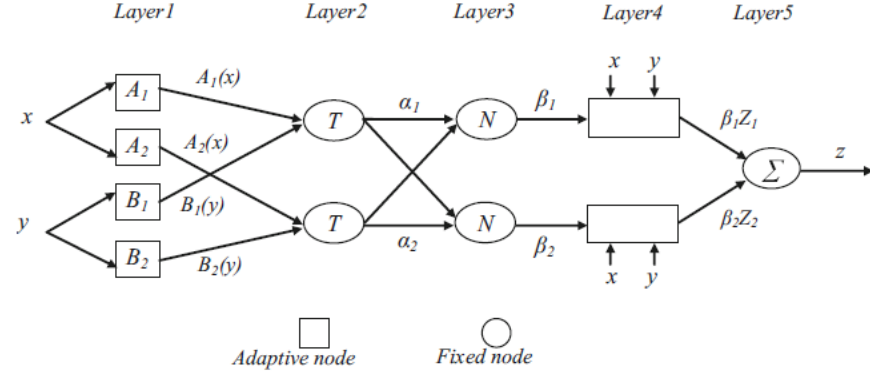

exhibiting various forms of membership functions on linguistic labels $A_{\mathrm{i}}$ and $B_{\mathrm{i}}$. The parameters in this layer are referred to as principle parameters.

Layer 2 Each node computes the firing strength of the associated rule. The nodes of this layer are called rule nodes. The outputs of the top and bottom neurons are as follows:

Top neuron

$\alpha_{1}=A_{1}(x) \times B_{1}(y)$

Bottom neuron

$\alpha_{2}=A_{2}(x) \times B_{2}(y)$

Layer 3 Every node in this layer is labeled by $\mathrm{N}$ to indicate the normalization of the firing levels. The output of the top and bottom neurons is normalized as follows:

Top neuron

$\beta_{1}=\alpha_{1} /\left(\alpha_{1}+\alpha_{2}\right)$

Bottom neuron

$\beta_{2}=\alpha_{2} /\left(\alpha_{1}+\alpha_{2}\right)$

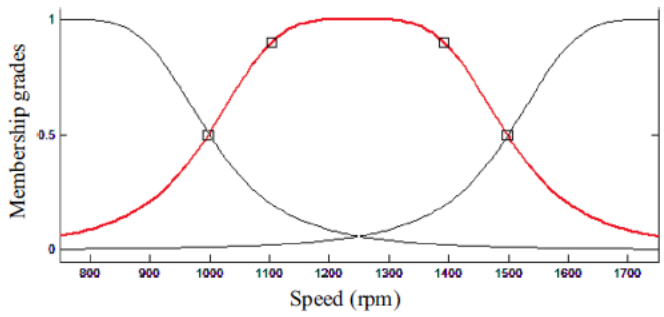

a) Initial membership function of speed
Layer 4 The output of the top and bottom neurons is the product of the normalized firing level and the individual rule output of the first rule and second rule, respectively.

Top neuron

$\beta_{1} z_{1}=\beta_{1}\left(a_{1} x+b_{1} y\right)$

\section{Bottom neuron}

$\beta_{2} z_{2}=\beta_{2}\left(a_{2} x+b_{2} y\right)$

Layer 5 The single node in this layer computes the overall system output as the sum of all incoming signals, i.e.,

$z=\beta_{1} z_{1}+\beta_{2} z_{2}$

If a crisp training set $\left(\left(x^{k}, y^{k}\right), k=1, \ldots, k\right)$ is given, then the parameters of the hybrid neural net (which determine the shape of the membership functions of the premises) can be learned by descent-type methods. The error function for pattern $k$ can be given by:

$E_{k}=\left(y^{k}-o^{k}\right)^{2}$

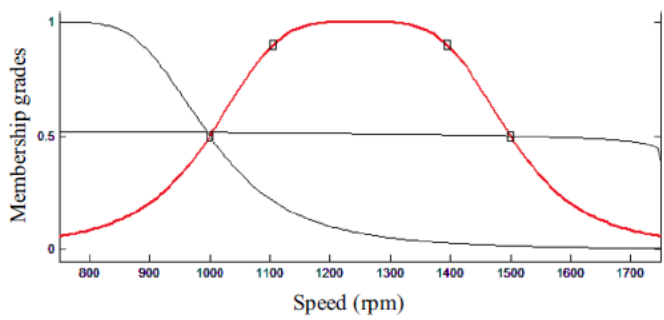

b) Final membership function of speed

Fig. 4 Initial and final membership function of speed. a Initial membership function of speed. b Final membership function of speed 


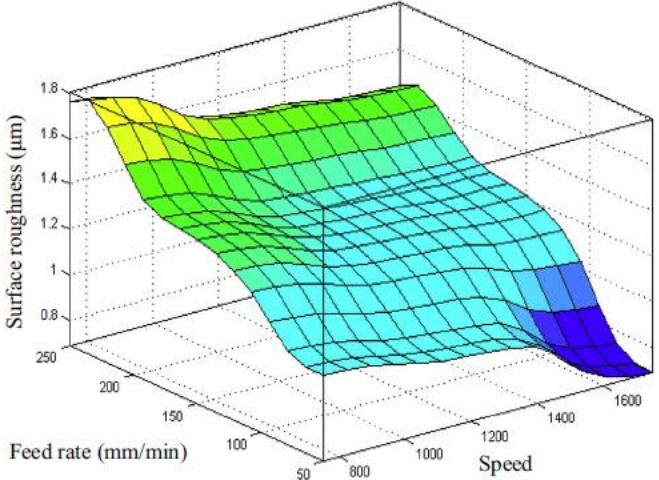

a) Variation of surface roughness with cutting parameters

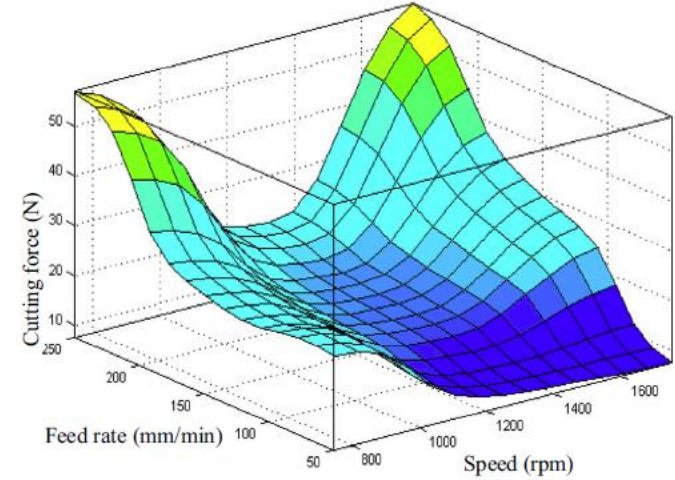

b) Variation of cutting force with cutting parameters

Fig. 5 The variation of surface roughness and cutting force with spindle speed and feed rate at $0.3-\mathrm{mm}$ depth of cut. a Variation of surface roughness with cutting parameters. $\mathbf{b}$ Variation of cutting force with cutting parameters

where $y^{\mathrm{k}}$ is the desired output and $o^{\mathrm{k}}$ is the computed output by the hybrid neural net [24].

\section{ANFIS prediction model results and discussion}

Figures 5, 6, 7, and 8 show the effects of the machining parameters on surface roughness and cutting force. In Fig. 5 at low depth of cut level $(0.3 \mathrm{~mm})$, it can be seen that the surface roughness decreases with increasing spindle speed and decreasing feed rate (Fig. 5a). This is because surface roughness is defined as the machining marks on the workpiece surface related to the geometry of the tool edge (Fig. 6 and Eq. 11) which is proportional to the feed rate [25, 26].

$$
R a=\frac{f_{z}^{2}}{t_{r} 18 \sqrt{3}} \quad\left(f_{z} \leq 2 t_{r} \sin (\psi)\right)
$$

Figure $5 \mathrm{~b}$ shows that the resultant cutting force decreases with increasing rotational speed at a low feed rate range (50 to $100 \mathrm{~mm} / \mathrm{min}$ ) for all ranges of cutting speed. But at feed rate ranging from 100 to $250 \mathrm{~mm} / \mathrm{min}$, the cutting force decreases with increasing rotational speed for speed ranging from 750 to $1350 \mathrm{rpm}$ and then the cutting force increases with increasing rotational speed for speed ranging from 1350 to $1750 \mathrm{rpm}$. This is mainly attributed to built-up edge formed at low speed, where the chip parts become a stationary body of highly deformed material attached to the cutting edge. The growth and rapid breakage of the built-up edge cause a rough surface on the machined part [27-29].
At medium and high depth of cut levels from 0.5 to $0.7 \mathrm{~mm}$ and feed rate ranging from 50 to $100 \mathrm{~mm} / \mathrm{min}$, the surface roughness and cutting force decrease with increasing rotational speed for all cutting speed ranges. At feed rate ranging from 100 to $250 \mathrm{~mm} / \mathrm{min}$, the surface roughness and cutting force decrease with increasing rotational speed for speed ranging from 750 to $1350 \mathrm{rpm}$; then the surface roughness and cutting force increase with increasing rotational speed for speed ranging from 1350 to $1750 \mathrm{rpm}$ as shown in Figs. 7 and 8.

From the above analysis, it can be seen that the cutting parameters against the cutting force show the same trend as the relations between cutting parameters and surface roughness. This has led to the conclusion that there is a strong correlation between the surface roughness and cutting force. Hence, it is possible to predict the curve trend of surface roughness from the cutting force.

Figure 9 shows the correlation between the cutting force and surface roughness. This figure indicates that surface roughness increases with increasing cutting force and vice versa. It is also clear that the trend of surface roughness change is steady at low cutting force ranging from 7 to $120 \mathrm{~N}$. However, for cutting ranges of more than $120 \mathrm{~N}$, surface roughness rapidly changes.

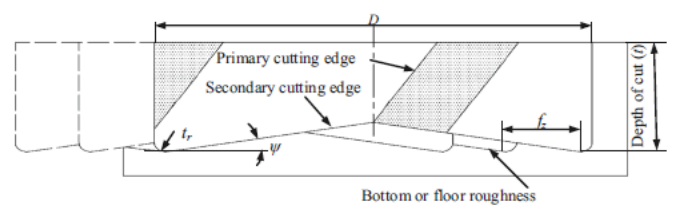

Fig. 6 Geometry of the tool edge in an end-milling

\section{Link to Full-Text Articles :}

